\title{
$\beta$-0xo Anilides in Heterocyclic Synthesis: Novel Synthesis of Substituted Pyridazinones, Pyridine, Pyrimidines and Pyrazolotriazines
}

\author{
Abdel Haleem Mostafa Hussein*, Mohamed Sayed Abdel Aal El-Gaby, \\ Ahmed Abdel Hameed Abd El-Maged, Ahmed Mohmed El-Sharief, Yousry Ahmed Ammar
}

Chemistry Department, Faculty of Science, Al-Azhar University, Assiut, Egypt

Email: ${ }^{*}$ abdelhaleemmh@yahoo.com

Received 3 April 2015; accepted 18 April 2015; published 23 April 2015

Copyright (C) 2015 by authors and OALib.

This work is licensed under the Creative Commons Attribution International License (CC BY). http://creativecommons.org/licenses/by/4.0/

(c) (i) Open Access

\begin{abstract}
Diazotization and coupling of acetoacetanilide derivative 1 with aromatic amines afforded the arylhydrazones 2a,b. Arylhydrazones 2a,b were treated with (DMF-DMA) to yield the pyridazine derivatives $3 a, b$ in good yield. Pyridazinone was treated with hydrazine hydrate to yield 4a,b. Reaction of 2a with hydroxylamine hydrochloride afforded the oxime derivative 5. Similarly, the reaction of acetoacetanilide 1 with hydroxylamine hydrochloride gave 3-hydroxyimino-N-p-tolylbutyramide 8 not the pyrazolone 9. The reaction of anilide 4 with aromatic aldehydes yielded $10 a, b$. Also, when anilide 4 was reacted with a mixture of aromatic aldehydes, urea or thiourea afforded the pyrimidines 11a,b. The reaction of anilide 1 with active methylene reagents was also investigated. So, 1 was reacted with malononitrile to give the pyridone derivative 12 . Similarly, anilide 1 was reacted with cyanoacetamide under the same reaction conditions to yield 4-methyl2-oxo-6-p-tolylamino-1,2-dihydro-pyridine-3-carbonitrile 13 in quantitative yield. The reaction of 1 with ylidenemalononitrile depends on structure of substituent. Thus, reaction of 1 with benzylidenemalononitrile or naphthylidenemalononitrile afforded 14a,b, while that with p-anisidinemalononitrile afforded 15. Coupling 1 with diazotized 16 - 18 afforded 19 - 21 respectively. Condensing 1 and aminopyrazoles $22 \mathrm{a}, \mathrm{b}$ afforded the acyclic adduct 23a,b rather than the pyrazolopyrimidine 24a,b.
\end{abstract}

\section{Keywords}

Heterocyclic Synthesis

Subject Areas: Analytical Chemistry, Organic Chemistry

\footnotetext{
${ }^{*}$ Corresponding author.
}

How to cite this paper: Hussein, A.M., El-Gaby, M.S.A., Abd El-Maged, A.A., El-Sharief, A.M. and Ammar, Y.A. (2015) 8-Oxo Anilides in Heterocyclic Synthesis: Novel Synthesis of Substituted Pyridazinones, Pyridine, Pyrimidines and Pyrazolotriazines. Open Access Library Journal, 2: e1439. http://dx.doi.org/10.4236/oalib.1101439 


\section{Introduction}

In the past few years, we have been involved in a program aimed at developing new efficient synthetic approaches for heteroaromatic compounds utilizing unexpensive starting materials [1] [2]. In continuation of our current interest in the syntheses of polyfunctionaly substituted heteroaromatic [3]-[6], we used here the readily obtainable $\beta$-oxo anilide derivatives as starting materials. It is worthwhile to explore their potential utility for the synthesis of polyfunctionaly substituted pyridazinones, pyridine, pyrimidines and pyrazolotriazines.

\section{Results and Discussion}

It has been observed that coupling of 3-oxo-N-p-tolyl-butyramide $\mathbf{1}$ with diazotized aromatic amines in ethanol buffered with sodium acetate at $0^{\circ} \mathrm{C}-5^{\circ} \mathrm{C}$ afforded the arylhydrazones 2a,b [7]. Arylhydrazones 2a,b were treated with N,N-dimethylformamide-dimethylacetal (DMF-DMA) in refluxing xylene to yield the pyridazinone derivatives $\mathbf{3 a}, \mathbf{b}$ in good yield. The structure $\mathbf{3}$ was confirmed bases on spectral data, elemental analysis and their chemical transformation. ${ }^{1} \mathrm{H}$ NMR spectrum for 3a revealed doublet at $\delta=6.9$ and 7.09 assigned for olefinic double bond $(\mathrm{J}=2.0 \mathrm{~Hz})$.

Compounds 3a,b were treated with hydrazine hydrate to yield a condensation product via water elimination as 4a,b. Compound 4a as example was established as the sole product based on elemental analysis and spectral data. Thus, IR spectrum showed absorption peaks at $v 3170,3380 \mathrm{~cm}^{-1}$ for $\mathrm{NH}_{2}$ group and disappearance of peaks at $v 1684 \mathrm{~cm}^{-1}$ for CO group.

Reactions of 2a with hydroxylamine hydrochloride in ethanolic solution containing amount of sodium acetate afforded the condensation product 5 that analyzed correctly for $\mathrm{C}_{17} \mathrm{H}_{18} \mathrm{~N}_{4} \mathrm{O}_{2}$. The structure of the latter product was identified as 2-(aryl-hydrazono)-3-hydroxyimino-N-p-tolyl-butyramide $\mathbf{5}$ on the basis of its IR and ${ }^{1} \mathrm{H}$ NMR spectra. The ${ }^{1} \mathrm{H}$ NMR spectrum displayed a two singlet signal at $\delta=9.59$ and $10.18 \mathrm{ppm}$ assigned to two $\mathrm{NH}$ group. Trials to cyclized compound 5 to triazole $\mathbf{6}$ or indazole $\mathbf{7}$ under different condition failed (Scheme $\mathbf{1}$ ).

Similarly, the reaction of $\beta$-Oxo anilide $\mathbf{1}$ with hydroxylamine hydrochloride in aqueous ethanol in presence of sodium acetate give 3-hydroxyimino-N-p-tolyl-butyramide 8 not the pyrazolone $\mathbf{9}$ [8]. Compound $\mathbf{9}$ was ruled out by spectroscopic data. The IR spectrum of oxime 8 revealed $v \mathrm{~cm}^{-1} 1652$ for amidic CO; 3174 for $\mathrm{NH} ; 3373$ for $\mathrm{OH}$.

The reaction of anilide $\mathbf{1}$ with aromatic aldehydes in the presence of ammonia has been reported to yield 2,6-dimethyl-1,4-dihydro-3,5-bis[(p-tolyl)carbamoyl]-4-(4-substituted phenyl)-pyridine 10a,b [9]. The structure of $\mathbf{1 0}$ was established bases on its correct elemental analysis and spectral data. Also, when compound $\mathbf{1}$ was reacted with a mixture of aromatic aldehydes and urea or thiourea, the tetrahydropyrimidines 11a,b was formed

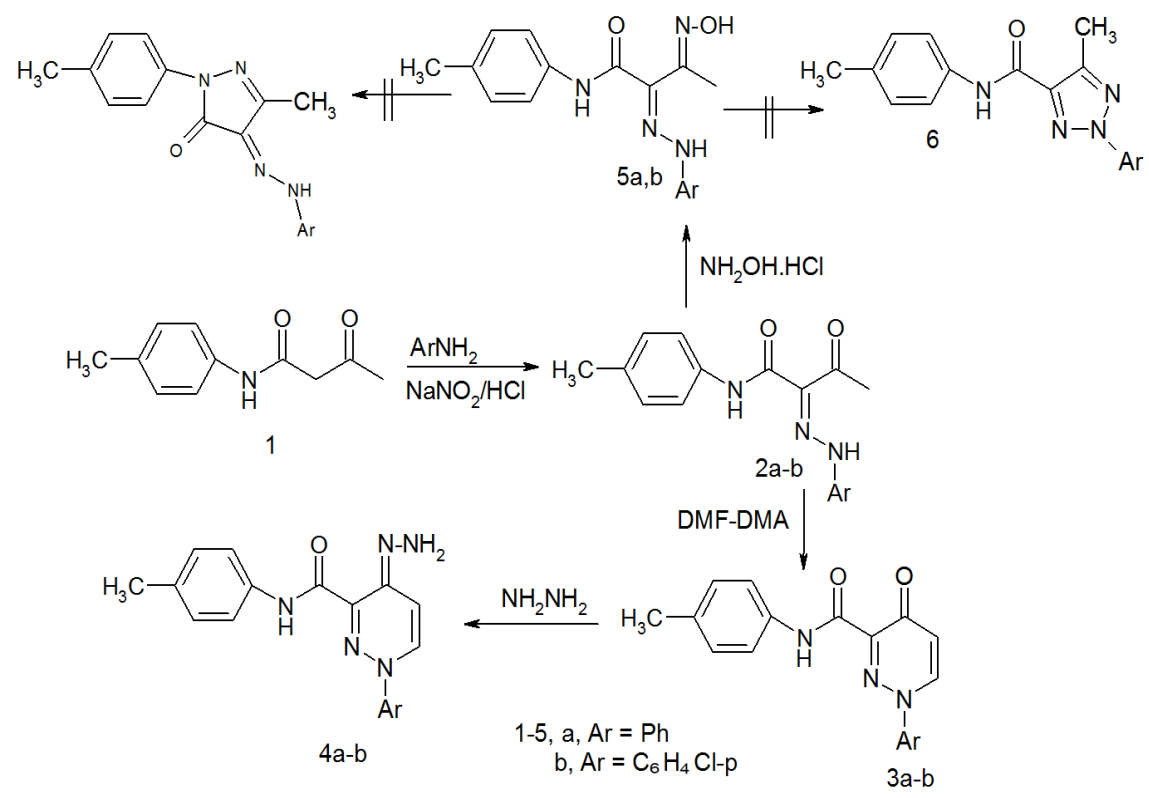

Scheme 1. Synthesis of azo compounds and pyridazine derivatives. 
[10]. The mass spectrum of $\mathbf{1 1 b}$ as example revealed a molecular ion peak at $\mathrm{m} / \mathrm{z}=371$ corresponding to the molecular formula $\mathrm{C}_{19} \mathrm{H}_{18} \mathrm{ClN}_{3} \mathrm{OS}$. Its ${ }^{1} \mathrm{H}$ NMR spectrum showed a singlet signal at $\delta=2.17$ and $2.28 \mathrm{ppm}$ assigned for the $2 \mathrm{CH}_{3}$ protons, a singlet signal $(1 \mathrm{H})$ at $\delta=5.47 \mathrm{ppm}$ assigned for pyrimidine- $4 \mathrm{H}$, a singlet at $\delta=$ 8.33, 9.22 and $9.97 \mathrm{ppm}$ assigned for 3NH protons (Scheme 2).

Reactions of compound $\mathbf{1}$ with active methylene reagents was also investigated. So, compound $\mathbf{1}$ was reacted with malononitrile in ethanolic piperidine to give the *yridine derivative $\mathbf{1 2}$. Structure $\mathbf{1 2}$ was supported by the appearance of $\mathrm{NH}_{2}$ absorption band at $v 3302,3466 \mathrm{~cm}^{-1}$ in the IR spectrum. Moreover, its ${ }^{1} \mathrm{H}$ NMR spectrum revealed a singlet signal at $\delta=5.6 \mathrm{ppm}$ assigned to $\mathrm{CH}$-pyridine and 6.61 assigned to $\mathrm{NH}_{2}$ in addition to the other functional group protons.

Similarly, compound 1 was reacted with cyanoacetamide in the same experimental conditions to give 4-methyl-2-oxo-6-p-tolylamino-1,2-dihydro-pyridine-3-carbonitrile $\mathbf{1 3}$ in quantitative yield.

The reaction of $\mathbf{1}$ with arylidenemalononitrile depends on structure of substituent. Thus, reactions of $\mathbf{1}$ with benzylidenemalononitrile or naphthylidenemalononitrile afforded $\mathbf{1 4 a , b}$ while with p-anisidinemalononitrile afforded 15. Structure 14 was established bases on its spectroscopic data. Thus, the IR spectrum of 14b for example revealed absorption bands at $v 3420$ and $3490 \mathrm{~cm}^{-1}$ assignable to $\mathrm{NH}_{2}$ group and $v 2245 \mathrm{~cm}^{-1}$ for $\mathrm{CN}$ group in addition to disappearance of band at $v 3260 \mathrm{~cm}^{-1}$ assignable to $\mathrm{NH}$ group. Assignment of structure $\mathbf{1 5}$ for the reaction product was based on its compatible spectroscopic data. Thus, its mass spectrum revealed a molecular ion peak at $m / z=373\left(\mathrm{M}^{+}\right)$corresponding to the molecular formula $\mathrm{C}_{22} \mathrm{H}_{19} \mathrm{~N}_{3} \mathrm{O}_{3}$. Its IR spectrum showed absorption band at $v 3210$ and $3300 \mathrm{~cm}^{-1}$ for (2NH), 2219 for (CN). Its ${ }^{1} \mathrm{H}$ NMR spectrum revealed a two singlet signal at $\delta=8.68,10.06 \mathrm{ppm}$ assigned for $2 \mathrm{NH}$ in addition to the other functional group protons (Scheme 3 ).

The active methylene group in compound $\mathbf{1}$ underwent an electrophilic substitution upon coupling with equimolar amounts of diazonium chloride of aminopyrazoles 16-18 afforded the expected pyrazolotriazines 19-21 respectively [11] [12]. These product compounds were established based on their elemental analysis and compatible spectroscopic data. Condensing 1 and aminopyrazoles 18b,c in refluxing isotropic water separator afforded the acyclic adduct 22a,b rather than the pyrazolopyrimidine 23a,b [13]. Assignment of structure 22 for the reaction product was based on its compatible spectroscopic data. Thus, its IR spectrum of 22a for example showed the presence of peak at $v 1665 \mathrm{~cm}^{-1}$ assigned for amidic carbonyl group (Scheme 4).

\section{Experimental}

All melting points are uncorrected. IR spectra $(\mathrm{KBr})$ were recorded on a Ft IR 5300 spectrometer $\left(v \mathrm{~cm}^{-1}\right)$. The ${ }^{1} \mathrm{H}$ NMR spectra were recorded in DMSO- $\mathrm{d}_{6}, \mathrm{CDCl}_{3}$ at $200-400 \mathrm{MHz}$ on a varian Gemini NMR spectrometer $(\delta$, ppm) using TMS as an internal standard. Mass spectra were obtained on GC Ms-QP 1000 EX mass spectrometer at 70 ev. Elemental analyses were carried out by the Microanalytical Research Center, Faculty of Science at Cairo University and Microanalytical Research Center at Assiut University.

\section{General procedure for preparation of compounds $2 a, b$.}

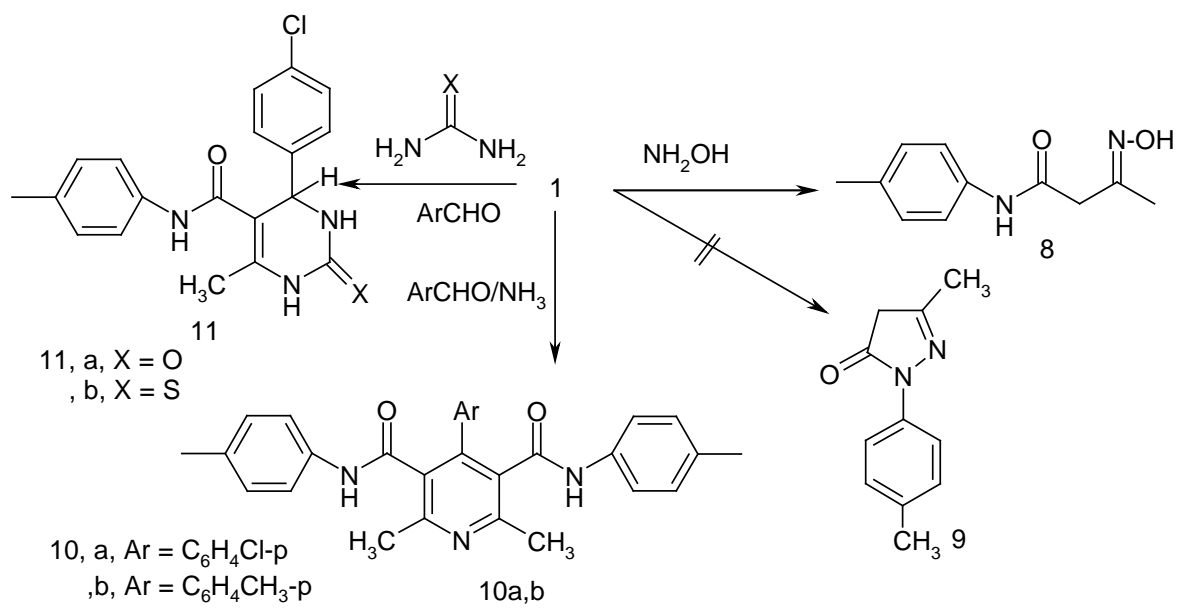

Scheme 2. Synthesis of pyridines and pyrimidines. 


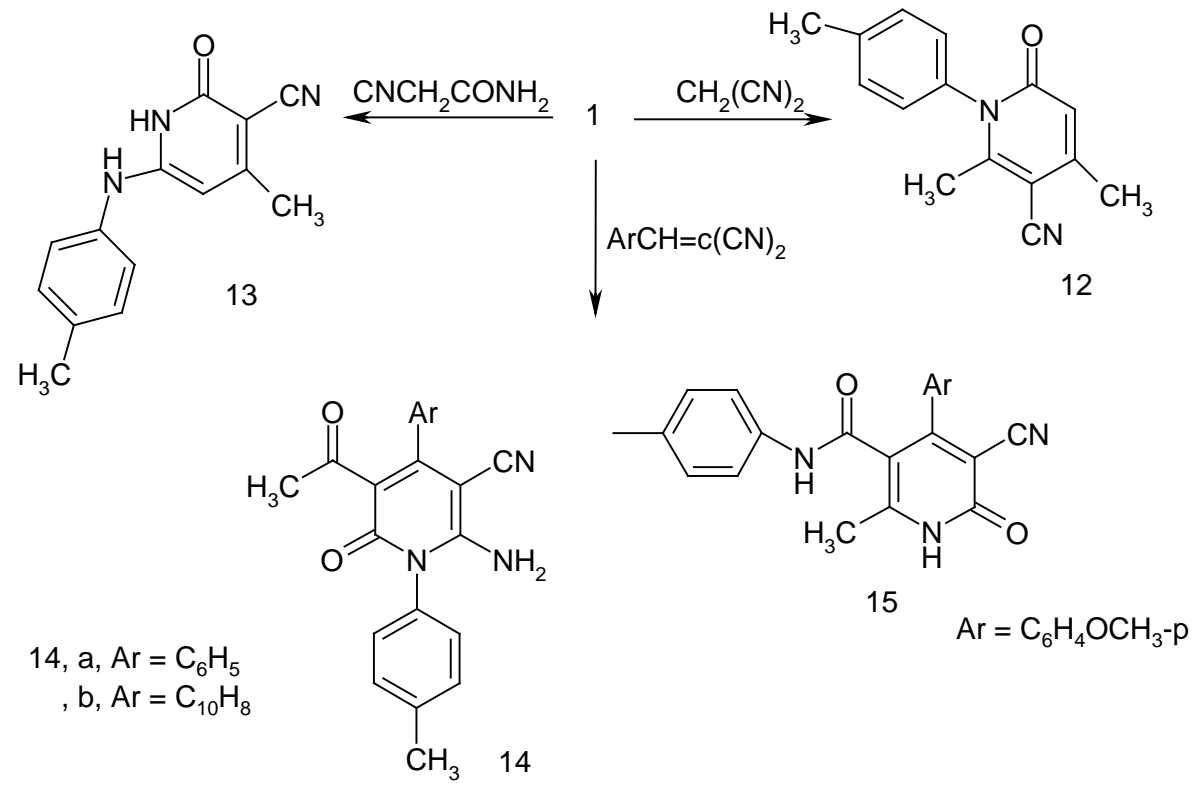

\section{Scheme 3. Synthesis of pyridine derivatives.}
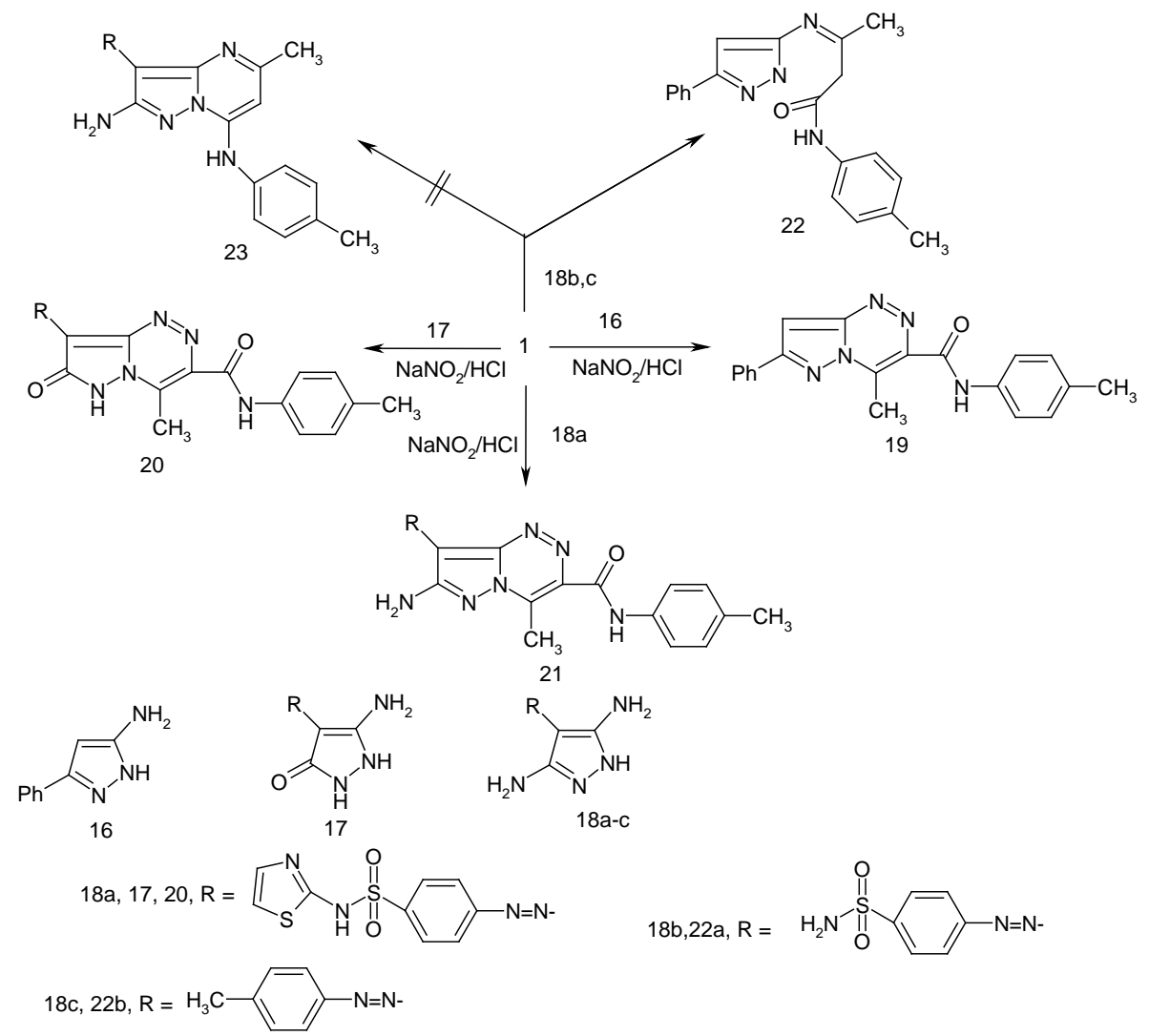

Scheme 4. Synthesis of pyrazolotriazine derivatives.

To a solution of compound 1 (0.01 mole) in dry xylene (10 ml), N,N-dimethylformamide-dimethylacetal ( 0.01 mole) was added. The reaction mixture was heated under reflux for $6 \mathrm{~h}$. The solvent was removed by evaporation under reduced pressure and the remainder was left to cool. The solid product so formed was collected 
by filtration, washed with petroleum ether (b.p. $40^{\circ} \mathrm{C}-60^{\circ} \mathrm{C}$ ) and the crude product recrystallized from ethanol to give $\mathbf{2 a , b}$.

4-Oxo-1-phenyl-3-(p-tolyl)carbamoyl-1,4-dihydro-pyridazine 2a.

This compound was obtained as brown crystals from ethanol; yield $72 \%$; mp. $175^{\circ} \mathrm{C}$. IR (KBr) $v \mathrm{~cm}^{-1} 1652$ (CO); 1684 (CO); 2983 (CH-aliph.); 3410 (NH). ${ }^{1} \mathrm{H}$ NMR $\left(\mathrm{CDCl}_{3}\right) \delta=2.27$ (s, 3H, $\mathrm{CH}_{3}$ ); 6.9 (d, 1H, CH); 7.09 (d, 1H, CH); 7.3-7.6 (m, 9H, Ar-H); 12.21 (s, 1H, NH). Found; C, 70.50; H, 4.70; N, 13.50; Calcd. For $\mathrm{C}_{18} \mathrm{H}_{15} \mathrm{~N}_{3} \mathrm{O}_{2}$ (305.33): C, 70.81; H, 4.95; N, 13.76\%.

\section{4-Oxo-1-(p-chloro phenyl)-3-(p-tolyl)carbamoyl-1,4-dihydro-pyridzine $2 \mathbf{b}$.}

This compound was obtained as brownish crystals from ethanol; yield $72 \%$; mp. $150^{\circ} \mathrm{C}$. IR $(\mathrm{KBr}) v \mathrm{~cm}^{-1} 1648$ (CO); 1680 (CO); 2982 (CH-aliph.); 3350 (NH). ${ }^{1} \mathrm{H}$ NMR $\left(\mathrm{CDCl}_{3}\right) \delta=1.27$ (s, 3H, $\left.\mathrm{CH}_{3}\right) ; 6.9$ (d, 1H, CH); 7.1-7.68 (m, 8H, Ar-H); 7.7 (d, 1H, CH); 11.34 (s, 1H, NH). Found; C, 63.50; H, 4.00; N, 12.10; Calcd. For $\mathrm{C}_{18} \mathrm{H}_{14} \mathrm{ClN}_{3} \mathrm{O}_{2}$ (339.78): C, 63.63; $\mathrm{H}, 4.15 ; \mathrm{N}, 12.37 \%$.

General procedure for preparation of compounds $3 a, b$.

A mixture of $\mathbf{2 a}, \mathbf{b}(0.01$ mole) and hydrazine hydrate $0.5 \mathrm{ml}$ in ethanol was refluxed for 6 hours. The product formed was collected and washed by cold ethanol to give $\mathbf{3 a , b}$ and recrystallized from proper solvent.

4-Hydrazono-1-phenyl-3-(p-tolyl)carbamoyl-1,4-dihydro-pyridazine 3 a.

This compound was obtained as greenish crystals from ethanol; yield $62 \% \mathrm{mp} .230^{\circ} \mathrm{C}$. IR (KBr) $v \mathrm{~cm}^{-1} 1648$ (CO); 2975 (CH-aliph.); 3170, $3380\left(\mathrm{NH}_{2}\right) .{ }^{1} \mathrm{H}$ NMR (DMSO-d $\left.\mathrm{d}_{6}\right) \delta=2.28$ (s, 3H, $\left.\mathrm{CH}_{3}\right) ; 7.05$ (d, 1H, CH); 7.92 (d, 1H, CH); 7.06-7.9 (m, 11H, Ar-H and $\mathrm{NH}_{2}$ ); 12.81 (s, 1H, NH). Found; C, 67.50; H, 5.20; N, 21.70; Calcd. For $\mathrm{C}_{18} \mathrm{H}_{17} \mathrm{~N}_{5} \mathrm{O}$ (319.36): C, 67.70; H, 5.37; N, 21.93\%.

1-(4-Chlorophenyl)-4-hydrazono-3-(p-tolyl)carbamoyl-1,4-dihydro-pyridazine $3 \mathrm{~b}$.

This compound was obtained as green crystals from ethanol; yield $62 \%$; mp. $220^{\circ} \mathrm{C}$. IR (KBr) $v \mathrm{~cm}^{-1} 1648$ (CO); 2968 (CH-aliph.); 3380, $3456\left(\mathrm{NH}_{2}\right)$. Found; C, 61.00; H, 4.40; N, 19.60; Calcd. For $\mathrm{C}_{18} \mathrm{H}_{16} \mathrm{ClN}_{5} \mathrm{O}$ (353.81): C, 61.11; H, 4.56; N, 19.79\%.

General procedure for preparation of compounds 5 and 8.

A mixture of $\mathbf{2 a , b}$ or $\mathbf{1}$ (0.01 mole), hydroxylamine hydrochloride and sodium acetate in ethanol was refluxed for 6 hours. The solid product so formed was collected on heating, washed with cold ethanol and recrystallized from proper solvent to give $\mathbf{5}$ and $\mathbf{8}$.

3-Hydroxyimino-2-(phenyl hydrazono)-N-p-tolyl-butyramide 5.

This compound was obtained as white crystals from ethanol, yield $65 \% \mathrm{mp} .170^{\circ} \mathrm{C}$. IR (KBr) $v \mathrm{~cm}^{-1} 1665$ (CO); 2925 (CH-aliph.); 3280, 3299 (2NH). ${ }^{1} \mathrm{H}$ NMR (DMSO-d 6 ) $\delta=1.53$ (s, 3H, $\mathrm{CH}_{3}$ ); 2.25 (s, 3H, $\mathrm{CH}_{3}$ ); 2.34 (s, 1H, OH); 7.10 - 7.51 (m, 9H, Ar-H); 9.59 (s, 1H, NH); 10.18 (s, 1H, NH). Found; C, 65.50; H, 5.60; N, 18.10; Calcd. For $\mathrm{C}_{17} \mathrm{H}_{18} \mathrm{~N}_{4} \mathrm{O}_{2}$ (310.36): C, 65.79; H, 5.85; N, 18.05\%.

3-Hydroxyimino-N-p-tolyl-butyramide 8.

This compound was obtained as white crystals from dioxane, yield $60 \% \mathrm{mp} .360^{\circ} \mathrm{C}$. IR (KBr) $v \mathrm{~cm}^{-1} 1652$ (CO); $3174(\mathrm{NH}) ; 3373(\mathrm{OH})$. Found; C, 64.10; H, 6.60; N, 13.50; Calcd. For $\mathrm{C}_{11} \mathrm{H}_{14} \mathrm{~N}_{2} \mathrm{O}_{2}$ (206.25): C, 64.06; $\mathrm{H}$, $6.60 ; \mathrm{N}, 13.50 \%$.

General procedure for preparation of compounds 10a,b.

A mixture of aldehyde ( 0.01 mole), compound $1\left(0.01\right.$ mole) and $0.7 \mathrm{ml} \mathrm{NH}_{3}(25 \% \mathrm{w} / \mathrm{v})$ were refluxed in 10 $\mathrm{ml}$ ethanol for 8-14 h. Cooled and then evaporated to dryness. The residue was crystallized from ethanol to give 10a,b.

4-(4-Chloro phenyl)-2,6-dimethyl-1,4-dihydro-3,5-bis[(p-tolyl)carba- moyl]-pyridine 10a.

This compound was obtained as white crystals, recrystallized from ethanol yield $70 \%$; mp. $220^{\circ} \mathrm{C}$. IR $(\mathrm{KBr}) v$ $\mathrm{cm}^{-1} 1686(\mathrm{CO}) ; 3285(\mathrm{NH}) .{ }^{1} \mathrm{H}$ NMR $\left(\mathrm{CDCl}_{3}\right) \delta=2.27$ (s, $\left.12 \mathrm{H}, 4 \mathrm{CH}_{3}\right) ; 7.0-7.41$ (m, 12H, Ar-H); 8.0 (s, 2H, $2 \mathrm{NH})$. Ms; $m / z=483\left(\mathrm{M}^{+}\right)$. Found; C, 71.60; H, 5.30; N, 8.50; Calcd. For $\mathrm{C}_{29} \mathrm{H}_{26} \mathrm{ClN}_{3} \mathrm{O}_{2}$ (484.00): C, 71.97; H, $5.41 ; \mathrm{N}, 8.68 \%$.

2,6-Dimethyl-1,4-dihydro-4-(p-tolyl)-3,5-bis[(p-tolyl)carbamoyl]-pyridine 10b.

This compound was obtained as white crystals, recrystallized from ethanol yield 70\%; mp. $195^{\circ} \mathrm{C}$. IR (KBr) $v$ $\mathrm{cm}^{-1} 1655$ (CO); 1699 (CO); 2925 (CH-aliph.); 3033 (CH-arom.); 3285 (NH). ${ }^{1} \mathrm{H}$ NMR (DMSO-d 6 ) $\delta=2.23$ (s,

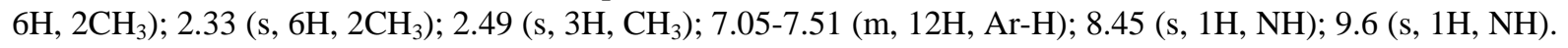
Found; C, 77.70; H, 6.30; N, 9.00; Calcd. For $\mathrm{C}_{30} \mathrm{H}_{29} \mathrm{~N}_{3} \mathrm{O}_{2}$ (463.58): C, 77.73; H, 6.31; N, 9.06\%.

General procedure for preparation of compounds 11a,b.

A mixture of compound $\mathbf{1}$ (0.01 mole), urea or thiourea ( 0.01 mole), p-Cl-benzaldehyde (0.01 mole), absolute 
ethanol (30 - $40 \mathrm{ml})$ and concentrated hydrochloric acid (8 - 10 drops) was stirred and slightly warmed on a steam bath till the mixture becomes a clear solution. It was allowed to stand overnight at ambient temperature. The product obtained was filtered off, dried and recrystallized from ethanol to give 11a,b.

4-(Chlorophenyl)-3,4-dihydro-6-methyl-5-(p-tolyl)carbamoyl-2(1H)-pyrimidinone 11a.

This compound was obtained as yellow crystals from ethanol; yield $80 \%$; mp. $295^{\circ} \mathrm{C}$. IR $(\mathrm{KBr}) v \mathrm{~cm}^{-1} 1638$ (CO); 1711 (CO); 3315, 3475 (2NH). ${ }^{1} \mathrm{H}$ NMR (DMSO-d $\left.\mathrm{d}_{6}\right) \delta=2.33$ (s, 3H, $\left.\mathrm{CH}_{3}\right) ; 2.52$ (s, 3H, CH $)$ ); 5.5 (s, $1 \mathrm{H}$, 4(H)-pyrimidine); 7.0 - 7.75 (m, 9H, Ar-H and NH); 8.81 (s, 1H, NH); 9.91 (s, 1H, NH). Found; C, 64.00; H, 5.30; N, 11.70; Calcd. For $\mathrm{C}_{19} \mathrm{H}_{18} \mathrm{ClN}_{3} \mathrm{O}_{2}$ (355.83): C, 64.14; H, 5.10; N, $11.81 \%$.

4-(Chlorophenyl)-3,4-dihydro-6-methyl-5-(p-tolyl)carbamoyl-2(1H)-pyrimidinethione 11b.

This compound was obtained as yellow crystals from ethanol; yield $78 \%$; mp. $180^{\circ} \mathrm{C}$. IR (KBr) $v \mathrm{~cm}^{-1} 1677$ (CO); 3275, 3456 (2NH). ${ }^{1} \mathrm{H}$ NMR $\left(\mathrm{CDCl}_{3}\right) \delta=2.17$ (s, 3H, $\left.\mathrm{CH}_{3}\right) ; 2.28\left(\mathrm{~s}, 3 \mathrm{H}, \mathrm{CH}_{3}\right)$; 5.47 (s, $1 \mathrm{H}, 4(\mathrm{H})$-pyrimidine); 7.0 - $7.42(\mathrm{~m}, 8 \mathrm{H}, \mathrm{Ar}-\mathrm{H}) ; 8.33(\mathrm{~s}, 1 \mathrm{H}, \mathrm{NH}) ; 9.22(\mathrm{~s}, 1 \mathrm{H}, \mathrm{NH}) ; 9.97$ (s, $1 \mathrm{H}, \mathrm{NH})$. Ms : $\mathrm{m} / \mathrm{z}=371\left(\mathrm{M}^{+}\right)$. Found; C, 61.20; H, 4.70; N, 11.20; Calcd. For $\mathrm{C}_{19} \mathrm{H}_{18} \mathrm{ClN}_{3} \mathrm{OS}$ (371.89): C, 61.37; H, 4.88; N, 11.30\%.

General procedure for preparation of compounds 12 and 13.

A mixture of 4 (0.01 mole), malononitrile (0.01 mole) and few drops of piperidine, was refluxed in ethanol for $4 \mathrm{~h}$. The obtained solid on heating recrystallized from ethanol to give 12 and $\mathbf{1 3 .}$

2-Amino-4-methyl-6-oxo-1-p-tolyl-1,6-dihydro-pyridine-3-carbonitrile 12.

This compound was obtained as white crystals from ethanol, yield $80 \% \mathrm{mp} .305^{\circ} \mathrm{C}$. IR (KBr) $v \mathrm{~cm}^{-1} 1668$ (CO); 2203 (CN); 3302, $3466\left(\mathrm{NH}_{2}\right) .{ }^{1} \mathrm{H}$ NMR $\left(\mathrm{DMSO}_{-}\right)$ CH-pyridine); 6.61 (s, 2H, NH ); 7.08 - 7.35 (m, 4H, Ar-H). Found; C, 70.00; H, 5.20; N, 17.30; Calcd. For $\mathrm{C}_{14} \mathrm{H}_{13} \mathrm{~N}_{3} \mathrm{O}$ (239.28): C, 70.28; H, 5.48; N, 17.56\%.

4-Methyl-2-oxo-6-p-tolylamino-1,2-dihydro-pyridine-3-carbonitrile 13.

This compound was obtained as white crystals from ethanol; yield 50\%; mp. $170^{\circ} \mathrm{C}$. IR $(\mathrm{KBr}) v \mathrm{~cm}^{-1} 1658$ (CO); 2217 (CN); 2951 (CH-aliph.); 3250, 3380 (2NH); Ms: m/z = 240 (M + 1). Found; C, 70.00; H, 5.20; N, 17.30; Calcd. For $\mathrm{C}_{14} \mathrm{H}_{13} \mathrm{~N}_{3} \mathrm{O}$ (239.28): C, 70.28; H, 5.48; N, 17.56\%.

General procedure for preparation of compounds 14a,b and 15.

To a solution of compound $\mathbf{1}(0.01 \mathrm{~mole})$ in ethanol ( $40 \mathrm{ml})$ containing a catalytic amount of piperidine ( 0.5 $\mathrm{ml})$, ylidenemalononitriles ( 0.01 mole) was added. The reaction mixture was heated under reflux for $6 \mathrm{~h}$. The solid product formed on heating was collected by filtration to give $\mathbf{1 4 a , b}$ and $\mathbf{1 5}$.

5-Acetyl-2-amino-6-oxo-4-phenyl-1-p-tolyl-1,6-dihydro-pyridine 14a.

This compound was obtained as yellow crystals; recrystallized from ethanol yield $80 \%$; mp. $202^{\circ} \mathrm{C}$. IR $(\mathrm{KBr})$ $v \mathrm{~cm}^{-1} 1648$ (CO); 1696 (CO); $2190(\mathrm{CN}) ; 3210,3280\left(\mathrm{NH}_{2}\right)$. Found; C, 73.30; H, 5.00; N, 12.00; Calcd. For $\mathrm{C}_{21} \mathrm{H}_{17} \mathrm{~N}_{3} \mathrm{O}_{2}$ (343.39): C, 73.45; H, 4.99; N, 12.24\%.

5-Acetyl-2-amino-4-naphthalen-1-yl-6-oxo-1-p-tolyl-1,6-dihydro-pyridine 14b.

This compound was obtained as yellow crystals from ethanol; yield $80 \%$; mp. $250^{\circ} \mathrm{C}$. IR (KBr) $v \mathrm{~cm}^{-1} 1632$ (CO); 1690 (CO); 2245 (CN); 3420, $3490\left(\mathrm{NH}_{2}\right) .{ }^{1} \mathrm{H}$ NMR $\left(\mathrm{CDCl}_{3}\right) \delta=1.91$ (s, 3H, $\left.\mathrm{CH}_{3}\right) ; 2.38$ (s, 3H, $\left.\mathrm{COCH}_{3}\right)$; 4.31 (broad, $2 \mathrm{H}, \mathrm{NH}_{2}$ ); 6.07 - 8.0 (m, 11H, Ar-H). Found; C, 76.10; H, 4.60; N, 10.50; Calcd. For $\mathrm{C}_{25} \mathrm{H}_{19} \mathrm{~N}_{3} \mathrm{O}_{2}$ (393.44): C, 76.32; H, 4.87; N, 10.68\%.

5-Cyano-4-(4-methoxyphenyl)-2-methyl-6-oxo-3-(p-tolyl)carbamoyl-1,6-dihydro-pyridine 15.

This compound was obtained as yellow crystals from dioxane; yield 72\%; mp. $200^{\circ} \mathrm{C}$. IR (KBr) $v \mathrm{~cm}^{-1} 1640$ (CO); 1692 (CO); 2219 (CN); 3210, 3300 (2NH). ${ }^{1} \mathrm{H}$ NMR (DMSO-d $\left.{ }_{6}\right) \delta=2.25$ (s, 3H, $\left.\mathrm{CH}_{3}\right) ; 2.48$ (s, 3H, $\mathrm{CH}_{3}$ ); 3.86 (s, 3H, $\mathrm{OCH}_{3}$ ); 6.9 - 7.42 (m, 8H, Ar-H); 8.68 (s, $\left.1 \mathrm{H}, \mathrm{NH}\right) ; 10.06$ (s, $\left.1 \mathrm{H}, \mathrm{NH}\right) . \mathrm{Ms}: \mathrm{m} / \mathrm{z}=373\left(\mathrm{M}^{+}\right)$. Found; C, 70.50; H, 5.00; N, 11.00; Calcd. For $\mathrm{C}_{22} \mathrm{H}_{19} \mathrm{~N}_{3} \mathrm{O}_{3}$ (373.42): C, 70.76; H, 5.13; N, 11.25\%.

General procedure for preparation of compounds 19-21.

A solution of 1 ( 0.01 mole $)$ in ethanol $(100 \mathrm{ml})$ containing sodium acetate $(2.0 \mathrm{~g})$ was cooled to $0^{\circ} \mathrm{C}$, stirred and treated gradually with a cooled solution of aryldiazonium chloride (prepared from 0.01 mole of amine 16-18 and the appropriate quantities of $\mathrm{HCl}$ and $\mathrm{NaNO}_{2}$ ). The solid product formed on standing was collected and recrystallized from the appropriate solvent to give 19-21.

4-Methyl-7-phenyl-3-(p-tolyl)carbamoyl-pyrazolo[5,1-c][1,2,4]-triazine 19.

This compound was obtained as brown crystals from ethanol yield $60 \% \mathrm{mp} .220^{\circ} \mathrm{C}$. IR (KBr) $v \mathrm{~cm}^{-1} 1670$ (CO); $3256(\mathrm{NH}) .{ }^{1} \mathrm{H}$ NMR $\left(\mathrm{CDCl}_{3}\right) \delta=1.27\left(\mathrm{~s}, 3 \mathrm{H}, \mathrm{CH}_{3}\right) ; 2.27\left(\mathrm{~s}, 3 \mathrm{H}, \mathrm{CH}_{3}\right) ; 7.06-7.61(\mathrm{~m}, 11 \mathrm{H}, \mathrm{Ar}-\mathrm{H}, \mathrm{CH}-$ pyrazole and NH). Found; C, 69.80; H, 4.90; N, 20.10; Calcd. For $\mathrm{C}_{20} \mathrm{H}_{17} \mathrm{~N}_{5} \mathrm{O}$ (343.37): C, 69.96; H, 4.99; N, $20.39 \%$. 


\section{4-Methyl-7-oxo-3-(p-tolyl)carbamoyl-8-[4-(thiazol-2-ylsulfamoyl)- phenyl-azo]-6,7-dihydro-pyrazolo} [5,1-c][1,2,4]triazine 20.

This compound was obtained as brownish crystals from ethanol; yield $70 \% \mathrm{mp} .220^{\circ} \mathrm{C}$. IR $(\mathrm{KBr}) v \mathrm{~cm}^{-1} 1640$ (CO); 1695 (CO); 2907 (CH-aliph.); 3104 (CH-arom.); 3160 (NH). Found; C, 50.80; H, 3.20; N, 22.70; Calcd. For $\mathrm{C}_{23} \mathrm{H}_{19} \mathrm{~N}_{9} \mathrm{O}_{4} \mathrm{~S}_{2}$ (549.59): C, 50.27; H, 3.48; N, 22.94\%.

7-Amino-4-methyl-3-(p-tolyl)carbamoyl-8-[4-(thiazol-2-ylsulfamoyl)-phenylazo]-pyrazolo[5,1-c][1,2,4]tr iazine 21.

This compound was obtained as brown crystals from dioxane; yield $60 \% \mathrm{mp} .300^{\circ} \mathrm{C}$. IR (KBr) $v \mathrm{~cm}^{-1} 1655$ (CO); 2925 (CH-aliph.); 3033 (CH-arom.); 3285, $3350\left(\mathrm{NH}_{2}\right) .{ }^{1} \mathrm{H}$ NMR (DMSO-d $\left.{ }_{6}\right) \delta=2.22$ (s, 3H, $\left.\mathrm{CH}_{3}\right) ; 2.28$ (s, $3 \mathrm{H}, \mathrm{CH}_{3}$ ); 6.8 - 7.81 (m, 14H, Ar-H, $\mathrm{CH}=\mathrm{CH}, 2 \mathrm{NH}$ and $\mathrm{NH}_{2}$ ). Found; C, 50.20; H, 3.50; N, 25.40; Calcd. For $\mathrm{C}_{23} \mathrm{H}_{20} \mathrm{~N}_{10} \mathrm{O}_{3} \mathrm{~S}_{2}$ (548.61): C, 50.36; H, 3.67; N, 25.53\%.

General procedure for preparation of compounds 22a,b.

A mixture of 18b,c (0.01 mole), compound $\mathbf{1}$ (0.01 mole), ammonium acetate in acetic acid/benzene (10/30 $\mathrm{ml}$ ) was heated under reflux (water separator) for $3 \mathrm{~h}$. The solvent was then evaporated under vacuo and the resulting solid products were filtered off and recrystallized from dioxane to give 23a,b.

3-[5-Amino-4-(4-sulfamoyl-phenylazo)-2H-pyrazol-3-ylimino]-N-p-tolyl-butyramide 22a.

This compound was obtained as brown crystals from dioxane; yield 55\%; mp. $308^{\circ} \mathrm{C}$. IR $(\mathrm{KBr}) v \mathrm{~cm}^{-1} 1665$ (CO); 3210, $3260\left(\mathrm{NH}_{2}\right) .{ }^{1} \mathrm{H}$ NMR $\left(\mathrm{DMSO}_{6}\right) \delta=1.90\left(\mathrm{~s}, 3 \mathrm{H}, \mathrm{CH}_{3}\right) ; 2.36\left(\mathrm{~s}, 3 \mathrm{H}, \mathrm{CH}_{3}\right) ; 5.82\left(\mathrm{~s}, 2 \mathrm{H}, \mathrm{CH}_{2}\right) ; 6.70$ (s, $2 \mathrm{H}, \mathrm{NH}_{2}$ ); 7.38 (s, 2H, NH ); 7.9-8.00 (m, 10H, Ar-H and 2NH). Found; C, 52.60; H, 4.60; N, 24.50; Calcd. For $\mathrm{C}_{20} \mathrm{H}_{22} \mathrm{~N}_{8} \mathrm{O}_{3} \mathrm{~S}$ (454.51): C, 52.85; H, 4.88; N, 24.65\%.

\section{3-(5-Amino-4-p-tolylazo-2H-pyrazol-3-ylimino)-N-p-tolyl-butyramide 22b.}

This compound was obtained as brown from dioxane crystals; yield 59\%; mp. $300^{\circ} \mathrm{C}$. IR $(\mathrm{KBr}) v \mathrm{~cm}^{-1} 1653$ (CO); $3150(\mathrm{NH})$; 3265, 3395 ( $\left.\mathrm{NH}_{2}\right)$. Found; C, 64.60; H, 5.70; N, 25.10; Calcd. For $\mathrm{C}_{21} \mathrm{H}_{23} \mathrm{~N}_{7} \mathrm{O}$ (389.46): C, 64.76; H, 5.95; N, 25.17\%.

\section{Conclusion}

We are successful to synthesize a novel substituted pyridazinones, pyridine, pyrimidines and pyrazolotriazines.

\section{References}

[1] G'Hussein, A.M. (1997) Studies with Polyfunctionally Substituted Heteroaromatics: A Facile Route for the Synthesis of Polyfunctionally Substituted N-Aminopyridines, 1,2,4-Triazolo[1,5-a]pyridines and Isoquinolines. Heteroatom Chemistry, 8, 1. http://dx.doi.org/10.1002/(SICI)1098-1071(1997)8:1<1::AID-HC1>3.0.CO;2-J

[2] Hussein, A.M. (1999) Afinidad, 484, 377.

[3] Elghandour. A.H.H., Hussein, A.M., Elnagdi, M.H. and Metwally, S.A. (1992) Polyfunctionally Substituted Heterocycles: Synthesis of New Polyfunctionally Substituted Phthalazines, Thieno[3,4-d]pyridazines and Pyrido[3,4-c]pyridazines. Journal für Praktische Chemie, 334, 723. http://dx.doi.org/10.1002/prac.19923340816

[4] Elnagdi, M.H., Elghandour, A.H.H., Harb, A.A., Hussein, A.M. and Metwally, S.A. (1992) Heterocycles, $38,739$.

[5] Hussein, A.M., Atalla, A.A. and Kamal El-Dean, A.M. (1995) Die Pharmazie, 50, 788.

[6] Hussein, A.M. (1998) Z. Naturforsch, 53b, 1.

[7] Hidehiro, T., Kensuke, A. and Koichi, T. (2004) Chem. Abs., 140, 254977.

[8] Hossbach, R., Lettabu, H., Nuhu, P., Schneider, R., Stenger, P. and Stiebitz, B. (1991) Pharmazie, 46, 412-415.

[9] Gelbard, G., Lin, J. and Roques, N. (1992) Reductions with NADH Models. 3. The High Reactivity of Hantzsch Amides. Journal of Organic Chemistry, 57, 1789-1793. http://dx.doi.org/10.1021/jo00032a035

[10] Sadanandam, Y.S., Shetty, M.M. and Diwan, P.V. (1992) Synthesis and Biological Evaluation of New 3,4-Dihydro6-methyl-5-N-methyl-carbamoyl-4-(substituted phenyl)-2(1H)pyrimidinones and Pyrimidinethiones. European Journal of Medicinal Chemistry, 27, 87-92. http://dx.doi.org/10.1016/0223-5234(92)90066-A

[11] Kaupp, G., Metwally, M.A., Fathy, A.A. and Abdel Latif, E. (2003) Quantitative Gas-Solid Diazotization of 3Aminopyrazolo[3,4-b]pyridine Derivatives and Azo Dye Syntheses by Means of Solid-Solid Reactions. European Journal of Organic Chemistry, 8, 1545-1551. http://dx.doi.org/10.1002/ejoc.200390213

[12] Ibrahim, M.K.A. (1989) Pakistan Journal of Scientific and Industrial Research, 32, 301-305.

[13] Abu Elmaati, T.M. and El-Taweel, F.M.A. (2002) Journal of the Chinese Chemical Society, 49, 1045-1050. 